University of South Carolina

Scholar Commons

Fall 2007

\title{
Toward a Population Health Model of Segmented Assimilation: The Case of Low Birth Weight in Los Angeles
}

\author{
Brian K. Finch \\ Nelson Lim \\ William Perez \\ D. Phuong Do \\ University of South Carolina - Columbia, phuongdo@mailbox.sc.edu
}

Follow this and additional works at: https://scholarcommons.sc.edu/

sph_health_services_policy_management_facpub

Part of the Public Health Commons

\section{Publication Info}

Published in Sociological Perspectives, Volume 50, Issue 3, Fall 2007, pages 445-468.

Finch, B. K., Lim, N., Perez, W., \& Do, D. P. (2007). Toward a population health model of segmented assimilation: The case of low birth weight in Los Angeles. Sociological Perspectives, 50(3), 445-468. DOI: $10.1525 /$ sop.2007.50.3.445

(C) Sociological Perspectives, 2007, University of California Press

This Article is brought to you by the Health Services Policy and Management at Scholar Commons. It has been accepted for inclusion in Faculty Publications by an authorized administrator of Scholar Commons. For more information, please contact digres@mailbox.sc.edu. 


\title{
TOWARD A POPULATION HEALTH MODEL OF SEGMENTED ASSIMILATION: THE CASE OF LOW BIRTH WEIGHT IN LOS ANGELES
}

\author{
BRIAN KARL FINCH \\ San Diego State University \\ NELSON LIM \\ RAND Corporation \\ WILLIAM PEREZ \\ Claremont Graduate University \\ D. PHUONG DO \\ University of Michigan
}

\begin{abstract}
The authors adapt the segmented assimilation theory to a model of population health, which posits that assimilation is actually harmful to migrants' health. The authors also specify models of individual and contextual factors to indirectly test the theory of segmented assimilationa theory that posits interactions between individual and residential circumstances. Using Year 2000 vital statistics data merged with 2000 U.S. census data from Los Angeles County, the authors model the probability of being born low birth weight among the native and foreign born. Results confirm an immigrant advantage at the individual level and protective effects of immigrant coresidence at the neighborhood level. Keywords: assimilation; birth weight; immigrant health
\end{abstract}

Throughout the history of the United States, each wave of immigrants has incited concerns of the newcomers' quality, their ability to assimilate, and the burden the country must bear to assimilate them into "mainstream" America. Leaving the definition of mainstream aside, the often unstated assumption in the discussion about immigration is that assimilation is beneficial for everyone, including the immigrants themselves, natives, and society. This is also the (often) unstated assumption related to the health and health burden of immigrants.

However, it has been shown that immigrants are relatively healthy, despite their inferior socioeconomic status (Markides and Coreil 1986), but this health

Address correspondence to: Brian Karl Finch, Professor of Sociology, San Diego State University, 5500 Campanile Drive, San Diego, CA 92182-4423; e-mail: bfinch@mail.sdsu.edu.

Sociological Perspectives, Vol. 50, Issue 3, pp. 445-468, ISSN 0731-1214, electronic ISSN 1533-8673.

(C) 2007 by Pacific Sociological Association. All rights reserved. Please direct all requests for permission to photocopy or reproduce article content through the University of California Press's Rights and Permissions website, at http://www.ucpressjournals.com/reprintinfo.asp. DOI: 10.1525/sop.2007.50.3.445. 
advantage declines over time (Vega and Amaro 1994). This is especially true for the case of birth outcomes in which immigrant women have healthy children and these offspring bear infants that are less healthy. This phenomenon implies that the acculturative process may be deleterious to immigrant health. Nonetheless, given our knowledge of the disparate assimilation patterns observed among various immigrant groups, the fate of health decline does not seem inevitable.

Therefore, it is our intention to test an application of segmented assimilation theory to birth weight - a major marker of population health-in an attempt uncover divergent pathways in health trajectories in a sample of Mexican American women. We use a unique data set that combines information from birth certificate data of a census of infants born in Los Angeles County in 2000 with information from the 2000 census, linked at the level of census tracts. We borrow from segmented assimilation theory to posit divergent health trajectories among immigrant women living in Los Angeles County.

\section{THE IMMIGRANT HEALTH PARADOX}

It has been fairly well established that immigrants have health profiles that belie their inferior socioeconomic status (Markides and Coreil 1986). Although most studies have focused on Latino subgroups, a handful of studies find that this advantage accrues to most immigrant groups (see, e.g., Frisbie, Cho, and Hummer 2001; Hummer, Biegler et al. 1999; Hummer, Rogers et al. 1999). In short, what is perceived to be a Hispanic paradox may be more accurately described as an immigrant paradox. ${ }^{1}$

The paradox of Hispanic health has its origin in Mexican immigration to the United States. Recent Mexican immigration to the United States has resulted in a Mexican American population with strong cultural ties to Mexico. In addition, Mexican Americans tend to be poorer, less educated, and medically underserved compared with non-Hispanic Whites. Despite these presumed socioeconomic risks, Mexican Americans are astonishingly healthy. The overall mortality rate among Mexican Americans is lower than that of non-Hispanic Whites (Sorlie et al. 1993), and Mexican American rates of infant mortality and low birth weight are equivalent to that of non-Hispanic Whites and are half that of Blacks (Collins and Shay 1994). This is a paradox of profound importance (Scribner 1996).

Two explanations may partly account for the favorable birth outcomes of immigrants. One is a cultural orientation that women from traditional societies bring to the host societies that encourages healthy behaviors that may be protective for birth outcomes (Guendelman 1998; Rumbaut and Weeks 1996; Scribner 1996). In an international comparative study, Guendelman et al. (1999) found that immigrant women were far less likely to smoke. Smoking was 17 times lower in Mexicoborn than U.S.-born women and 2.6 times lower in North African than French women. Furthermore, among the smokers, immigrants consumed fewer cigarettes than did natives/nationals. Other studies have found a low rate of addictive substance use, such as tobacco, alcohol, and illicit drugs, by Mexico-born women in the United States and North African women in France (Guendelman 1998; Wanner, Khlat, and Bouchardy 1995). 
Another explanation for the favorable birth outcomes of immigrants may be the healthy immigrant effect, which argues that immigrants may compose a selected group who are healthier than their compatriots who do not migrate (Marmot, Adelstein, and Bulusu 1984). For instance, whereas 5.3 percent of infants of Mexicoborn women in the United States were low birth weight, the estimated rate in Mexico in 1990 was 12 percent (UNICEF 1996).

Each of these studies suggests that although immigrants may experience health advantages at the time of migration, this advantage gradually erodes, on average. However, given what we know about divergent assimilation trajectories, it is very possible that health trajectories may follow similar pathways, and we turn to a relevant theory for assistance in hypothesizing plausible divergences.

\section{SEGMENTED ASSIMILATION THEORY AND HEALTH}

Addressing the divergent processes by which individual immigrants experience socioeconomic mobility (upward or downward), the theory of segmented assimilation lends itself particularly well to understanding the processes of adaptation among immigrants. ${ }^{2}$ In addition to socioeconomic consequences, we contend that these processes consist of adaptation and assimilation to cultural practices (e.g., diet/nutrition, experiences of stress, maintenance of support networks, risk behaviors such as substance use) that may have important implications for health as well.

According to the traditional understanding of the segmented assimilation theory, new immigrants and their children face three possible paths to assimilation (Portes and Zhou 1993; see also Portes and Rumbaut 2001: 44-69). First, some immigrants, especially those with high levels of education, will be able to replicate the well-traveled path of European immigrants of the last great immigration of the nineteenth century (Alba and Nee 1999; Card, DiNardo, and Estes 2000). Over successive generations, they will integrate into the economy and acculturate into mainstream society. Second, newcomers may find barriers insurmountable and come to share the fate of the urban underclass instead. Third, immigrants may find refuge in their ethnic communities and find ways to assimilate into mainstream society, without falling victim to the cultural and structural constraints of the urban underclass. The segmented assimilation models look to the immigrant culture and social solidarity as critical factors in the assimilation process.

To date, most tests of the segmented assimilation hypothesis relate to socioeconomic attainment. Nonetheless, the theory is clearly defined in terms of cultural adaptation and the socialization processes that immigrants undertake. As such, it provides a general framework for the ways in which individual immigrants come to pursue and experience differential levels of integration into American life-the paradox being that assimilation is not without its "discontents" (Rumbaut 1997). For this reason, we contend that the theory of assimilation also lends itself well to explaining divergent health outcomes among immigrants.

With respect to health outcomes, however, the consequences of cultural adaptation do not appear to follow the same path as occupational or socioeconomic 
mobility. In fact, adaptation to mainstream American culture among immigrants tends to result in the adoption of poor health behaviors and health deterioration (Rumbaut 1997). Acculturation and integration into either middle-class America or the urban underclass may both have negative health implications for immigrants, regardless of one's potential socioeconomic attainment and predicted mobility. In this context, it is not the destination of assimilation but the process of assimilation itself that may have a negative impact on immigrants' health.

Accordingly, we adapt the theory of segmented assimilation-in which assimilation is construed as deleterious to health-and posit three divergent pathways that immigrants might follow after immigration to the United States, and we assess the applicability of this theory to health outcomes in general. We then apply this theory in developing and testing our hypotheses, using birth weight as our health outcome of interest.

\section{THE SIGNIFICANCE OF BIRTH WEIGHT AS A HEALTH MEASURE}

We select low birth weight as our key outcome to test our hypotheses because it has several useful analytical properties and because it is such a crucial health indicator of both current health and mortality risk and life course health trajectories. First, reverse causation is minimized as infant health does not determine the acculturation status or residential context of mothers. Second, birth weight is a fairly accurately measured health indicator that is not subject to the bias that other indicators of health among immigrant populations are. For example, self-reported health is thought to be artifactually related to acculturation (Angel and Guarnaccia 1989; Finch et al. 2002), and passive studies of mortality are subject to return migration and may bias studies of mortality (Palloni and Morenoff 2002).

Additionally, the selection of low birth weight as a particularly important health outcome is crucial for several well-established reasons. First, low birth weight is strongly associated with the risk of infant mortality, particularly in the neonatal period (Gortmaker and Wise 1997). Second, low-birth-weight infants are at higher risk for several crucial developmental and health outcomes, including cognitive development (Hack, Klein, and Taylor 1995), school difficulty and hyperactivity (McCormick, Gortmaker, and Sobol 1990), and a higher prevalence of respiratory distress and asthma (Boardman, Finch, and Hummer 2001), just to name a few. In addition, it has been argued and documented that the disadvantages of adverse birth outcomes, such as low birth weight, persist into late adolescence and adulthood (Barker 1995; Boardman et al. 2002; Elo and Preston 1992).

\section{HYPOTHESES}

From a public health perspective, researchers find that the longer immigrants have lived in the United States, the more maladaptive the outcomes are (see, e.g., Harker 2001; Rumbaut and Ima 1988). As immigrant groups become more highly assimilated into mainstream American values and customs, changes in healthrelated attitudes and behaviors also may occur. Acceptance of the predominant 
values may make such a group more amenable to the prevailing social norms of health behaviors. The pattern of diseases characterizing the group may also shift toward that experienced by the majority group.

Immigrants who settle into neighborhoods where their compatriots live, however, have significant advantages over those who try to find their way among strangers. "Such communities can cushion the impact of a foreign culture and provide assistance for finding jobs. Help with immediate living needs, such as housing, places to shop, and schools for the children, also flow through these coethnic networks" (Portes and Rumbaut 2001: 48).

Within this framework, we can effectively test hypotheses proposed by our adapted segmented assimilation thesis, which contends that mainstream adaptation may be deleterious to health (while recognizing that adaptation may be simultaneously beneficial for other outcomes, such as upward economic mobility) and that underclass adaptation may also be deleterious to health. We specify three separate groupings of hypotheses concerning the relationship between nativity, neighborhood conditions, and occupational status and health outcomes: (a) immigrants living near an urban underclass are at an increased risk for assimilating into a local subculture, as indicated by negative health outcomes; (b) immigrants living in an immigrant community are subject to sociocultural resources that foster the protective factors that lead to positive health outcomes, such as better diets, less substance abuse, and better mental health, among first-generation immigrants; (c) compared to immigrants living in or near an urban underclass population, those who live in an ethnic community are more likely to have more positive health outcomes. In other words, neighborhood-level risk factors, preventive effects of living under strong informal ethnic kinship social networks, and the interaction between these two sets of contextual factors can explain divergent health outcomes among immigrants.

First, we hypothesize that we will observe an immigrant health advantage at the individual level (Hypothesis 1a) such that infants of foreign-born mothers will be less likely to be born with low birth weight. In addition, we expect that infants born to mothers living in immigrant neighborhoods will be less likely to be born low birth weight (Hypothesis $1 \mathrm{~b}$ ). We also expect to find the previously observed relationship between neighborhood disadvantage and risk of low birth weight (Hypothesis 1c).

Second, we hypothesize that the effects of neighborhood conditions will moderate the relationship between nativity and low birth weight. In particular, we expect that the effects of immigrant neighborhoods will be more protective of infants of foreign-born mothers (Hypothesis 2a), whereas the effects of neighborhood disadvantage will be heightened among the native born (Hypothesis $2 b$ ).

Third, we hypothesize that predicted risks for professional migrants will be higher than for those of labor migrants (Hypothesis 3a) and that immigrant neighborhoods will be more protective of infants of foreign-born mothers than they will be for infants of native-born and professional migrants (Hypothesis $3 b)$. Furthermore, we expect that neighborhood disadvantage will have heightened deleterious effects on professional migrants and the native born (Hypothesis 3c). 


\section{SUMMARY OF HYPOTHESES}

Hypothesis 1: Both individual and contextual factors are related to low birth weight.

Hypothesis 1a: At the individual level, nativity confers a health advantage such that infants of foreign-born mothers will be less likely to be born with low birth weight.

Hypothesis $1 b$ : Infants born to mothers living in unacculturated neighborhoods will be less likely to be born with low birth weight.

Hypothesis 1c: Neighborhood disadvantage will be positively related to the risk of low birth weight.

Hypothesis 2: The effects of neighborhood conditions will moderate the relationship between nativity and low birth weight.

Hypothesis $2 a$ : The effects of unacculturated neighborhoods will be more protective of infants of foreign-born mothers.

Hypothesis $2 b$ : The effects of neighborhood disadvantage will be heightened among the native born.

Hypothesis 3: The risk of low birth weight depends on the occupational status of migrants.

Hypothesis $3 a$ : The predicted risks of low birth weight will be higher for professional migrants than for labor migrants.

Hypothesis 3b: Unacculturated neighborhoods will be more protective of infants of foreign-born mothers than of infants of native-born and professional migrants.

Hypothesis 3c: Neighborhood disadvantage will have heightened deleterious effects on professional migrants and the native born.

\section{DATA AND METHODS}

\section{Data}

We use two data sets to conduct our analyses. First, we contacted the Los Angeles County Department of Health Services and received a vital records data file for all births occurring to residents of Los Angeles County $(N=165,932)$ in 2000. ${ }^{3}$ A separate county agency (the Data Collection and Analysis Unit), relying on the most recent address files, was able to accurately geo-code maternal addresses into valid census tract codes for more than 97 percent of the birth mothers in the data. ${ }^{4}$ Second, we merged data from the 2000 U.S. Census to each case in the vital records birth file at the census tract level. Although census tracts are administratively defined boundaries, their borders are drawn with respect to maintaining sociodemographic homogeneity and geographic consistency. 
We excluded all cases for which a census tract was unknown or incorrectly recorded, there were multiple births, and there were extreme birth weights and gestation lengths that are largely due to recording errors, resulting in the final analysis sample size of 140,472 infants. ${ }^{5}$ These infants were born in 2,035 of the 2,052 populated census tracts in Los Angeles County. In addition, there were between 3 and 83 births per tract in our analysis file, with an average of 39.68 births per tract (median $=42$ ). Census tracts in Los Angeles County represent, on average, an area of 2.5 square miles and contain just less than 6,000 people per tract $(M=5,996)$.

\section{Individual-Level Variables ${ }^{6}$}

Our key dependent variable is a measure of low birth weight $(<2,500 \text { grams })^{7}$; just less than 5 percent ( 4.88 percent) of infants born in Los Angeles County in 2000 were low birth weight. This is the only variable, other than infant sex, measured at the infant level; the remainder of the individual-level variables rely on maternal reports and clinical records recorded in the birth certificate (see Table 1 for descriptive statistics). Our key predictor variable is nativity. As nativity may broadly subsume several characteristics of individuals, such as primary language spoken, cultural attachment and affinity, country of primary education, cultural norms and practices, the presence of stronger social support networks, ethnic identity, and the effects of health selection (i.e., that immigrants are healthier than residents of their origin population), we operationalize nativity in two ways. In the first case, nativity is recorded as mothers born in the United States (i.e., native born) versus mothers born in all other countries (i.e., foreign born)—while a second type separates out labor migrants from professional migrants. This is accomplished by splitting the group of foreign-born mothers into those who have at least sixteen years of education (professional migrants) and those with fifteen years or less (labor migrants).

Mother's age is represented by a term, raising the linear age (twelve to fiftyfour) to a half-power (i.e., the square root) and including this term simultaneously with a raw age variable (i.e., Age $^{5}$ and Age). This bowl-shaped function was curvilinear such that increasing age from twelve to twenty-two resulted in declines in low-birth-weight risk with virtually no change in risk from age twenty-two to thirty-two and an increase in risk for women older than thirty-three. Next, parity is defined as an interaction between age and number of births, as defined by Kleinman and Kessel (1987); this variable is only nominally collinear with age but does not affect the model or the estimate of our key variables. This variable is categorized into primiparous births, high parity, and low parity. ${ }^{8}$ Marital status is represented by a dummy variable indicating married versus unmarried, and race/ethnicity is represented by sets of dummy variables for Latino, non-Hispanic White, non-Hispanic Black, non-Hispanic Asian, and Other.

Socioeconomic status is represented by several variables, including maternal education, the primary payer for the birth, and adequacy of prenatal care. Mother's education is specified in the regression models by a second-order fractional polynomial (similar to age) in which cubic education is included simultaneously with cubic education multiplied by the natural logarithm of raw education. This 
TABLE 1

Descriptive Statistics for Model Variables

\begin{tabular}{|c|c|c|c|c|c|c|}
\hline Variables & $\mathrm{N}$ & $\%$ & $M$ & $S D$ & Range & $\%$ LBWT \\
\hline \multicolumn{7}{|l|}{ Neighborhood-level variables } \\
\hline $\begin{array}{l}\text { Neighborhood } \\
\text { disadvantage }\end{array}$ & & & 14.79 & 6.96 & 0 to 100.00 & \\
\hline Immigrant orientation & & & 28.16 & 13.61 & 0 to 71.45 & \\
\hline \multicolumn{7}{|l|}{ Individual-level variables } \\
\hline Nativity [native born] & 60,493 & 43.06 & & & & 5.45 \\
\hline Foreign born & 79,687 & 56.73 & & & & 4.44 \\
\hline Missing & 292 & 0.21 & & & & 5.02 \\
\hline Nativity [native born] & 60,493 & 43.15 & & & & 5.45 \\
\hline Labor migrant & 68,535 & 48.89 & & & & 4.39 \\
\hline Professional migrant & 11,152 & 7.96 & & & & 4.77 \\
\hline Missing & 292 & 0.21 & & & & 5.02 \\
\hline Mother's age & & & 27.66 & 6.32 & 12 to 54 & \\
\hline Parity [first birth] & 53,463 & 38.06 & & & & 5.85 \\
\hline Low parity & 76,159 & 54.22 & & & & 4.32 \\
\hline High parity & 10,814 & 7.70 & & & & 4.32 \\
\hline Missing & 36 & 0.03 & & & & 2.78 \\
\hline $\begin{array}{l}\text { Marital status } \\
\text { [unmarried] }\end{array}$ & 51,544 & 36.69 & & & & 5.52 \\
\hline Married & 88,928 & 63.31 & & & & 4.52 \\
\hline $\begin{array}{l}\text { Race/ethnicity [White, } \\
\text { non-Hispanic] }\end{array}$ & 25,574 & 18.21 & & & & 3.89 \\
\hline Latino & 87,976 & 62.23 & & & & 4.50 \\
\hline Black, non-Hispanic & 11,706 & 8.33 & & & & 9.41 \\
\hline Asian, non-Hispanic & 14,867 & 10.58 & & & & 5.28 \\
\hline Other & 133 & 0.10 & & & & 5.63 \\
\hline Mother's education & & & 11.79 & 3.36 & 0 to 17 & \\
\hline $\begin{array}{l}\text { Payer for birth [private } \\
\text { insurance] }\end{array}$ & 67,533 & 48.08 & & & & 4.58 \\
\hline Government & 69,444 & 49.44 & & & & 5.12 \\
\hline Self-pay & 3,185 & 2.27 & & & & 6.15 \\
\hline Other & 306 & 0.22 & & & & 6.86 \\
\hline Prenatal care [adequate] & 60,385 & 42.99 & & & & 2.77 \\
\hline Inadequate & 11,422 & 8.13 & & & & 5.54 \\
\hline Intermediate & 20,591 & 14.66 & & & & 2.67 \\
\hline Adequate-plus & 43,243 & 30.78 & & & & 8.77 \\
\hline Missing/no care & 4,831 & 3.44 & & & & 4.49 \\
\hline Sex of child [female] & 68,515 & 48.77 & & & & 5.12 \\
\hline Male & 71,457 & 51.26 & & & & 4.67 \\
\hline Total & 140,472 & 100.00 & & & & 4.88 \\
\hline
\end{tabular}

Note: LBWT = low birth weight. 
reverse s-shaped function yields a monotonic decline across the distribution for the risk of being low birth weight with flatter sections between zero to five years of education and between twelve or more years of education. In other words, the largest negative slope in education effects occurs between five and twelve years of education. Payer-for-birth categories include private insurance, government (e.g., Medicaid, Medical), self-pay, and other. The Kotelchuck Adequacy of Prenatal Care Utilization indicates prenatal care usage-an index that accounts for the observed negative effects of medically mandated overuse (Kotelchuck 1994). These categories include inadequate care, intermediate care, adequate care, adequate-plus care, and missing/no care. Finally, the sex of the child is included as a dummy for male infants; this is included because of the increased risk of female infants being born low birth weight in spite of the fact that infant sex is generally orthogonal to other determinants of low-birth-weight status.

Table 1 indicates that this is not a typical sample of births nationwide. Given that this is a census of births in Los Angeles, 56 percent of the infants are born to foreign-born mothers, and nearly two-thirds of the total births are to Hispanic mothers. However, despite the relative disadvantage that is observed in Table 1 (e.g., average education is some high school, nearly 50 percent of the births are being paid for from government sources), only 4.9 percent of infants are born low birth weight, well below the national average for 2000 (around 7.6 percent).

\section{Contextual-Level Variables}

Residential context may reflect both potential shocks to health because of both the material conditions of a neighborhood (e.g., propensity for crime, lack of capital investment, stressful living conditions) and the cultural context of the aggregate of residents (e.g., immigrant enclaves or urban underclass conditions). For example, foreign-born individuals may be able to draw on the resources available in immigrant enclaves because they are more likely to share a common language. Conversely, foreign-born individuals may be more resistant to the pressures of urban underclass living either because of a more supportive home environment or because of a general propensity to resist acculturation to nonnormative patterns of behavior.

We selected several variables to represent two constructs at the neighborhood (census tract) level: neighborhood disadvantage and neighborhood immigrant orientation. Four variables were averaged to create a scale of neighborhood disadvantage, including the percentage of persons in the census tract in poverty, the percentage of households receiving public assistance, the percentage of femaleheaded households, and the percentage of unemployed males. ${ }^{9}$ These four items were highly correlated and yielded a Cronbach's alpha (scale reliability coefficient) of .8468 . This scale ranges from 0 to 100 with a mean of around 15 and a standard deviation of around 7; high values represent more disadvantage in the neighborhood. In addition, immigrant orientation was captured by three measures, including the percentage of persons in the tract who were foreign born, the percentage of noncitizens in a tract, and the percentage of tract households who are linguistically isolated, with higher values on this scale representing more immigrant orientation in the neighborhood. ${ }^{10}$ These three items also correlate highly together and yield an alpha of .9663. Descriptive statistics for the scales and 
TABLE 2

Index Items for Scales of Neighborhood Disadvantage and Neighborhood Immigrant Orientation

\begin{tabular}{lcrrc}
\hline Construct/Variables & Cronbach's $\alpha$ & \multicolumn{1}{c}{$M$} & \multicolumn{1}{c}{$S D$} & Range \\
\hline Neighborhood disadvantage & .8468 & 14.79 & 6.96 & 0.00 to 100.00 \\
$\quad$ Percent persons in poverty & & 20.72 & 12.29 & 0.00 to 100.00 \\
$\quad$ Percent persons receiving public & & 8.55 & 6.20 & 0.00 to 49.06 \\
$\quad$ assistance income & & & & \\
$\quad$ Percent female-headed households & & 20.98 & 8.36 & 0.00 to 72.32 \\
Percent males unemployed & & 8.90 & 4.74 & 0.00 to 100.00 \\
Immigrant orientation & .9663 & 28.16 & 13.61 & 0.00 to 71.45 \\
$\quad$ Percent persons foreign born & & 39.26 & 15.16 & 0.00 to 79.10 \\
$\quad$ Percent persons noncitizens & & 25.69 & 13.62 & 0.00 to 66.90 \\
Percent households linguistically & & 19.54 & 13.36 & 0.00 to 100.00 \\
$\quad$ isolated & & & & \\
\hline
\end{tabular}

Note: These are computed for all 2,052 populated census tracts in Los Angeles County.

individual items are presented in Table 2. This scale ranges from 0 to 74 with a mean of 28 and a standard deviation of 14; higher values represent a greater immigrant orientation in the neighborhood. Immigrant orientation and disadvantage are also moderately positively related $(r=.5169)$, so simultaneous controls are necessary to isolate any independent effects. However, colinearity did not appear to alter the results when both contextual-level indexes are modeled simultaneously.

From the raw scales for these two variables, we employed a covariate-adjusted fractional polynomial approach to determine the best functional form. The form for each contextual predictor is as follows: (a) neighborhood disadvantage, $\ln$ (Disadvantage), and (b) neighborhood immigration orientation ${ }^{3}$, and $\ln$ (immigrant orientation) ${ }^{3}$. The use of log-linear neighborhood disadvantage was simple, but the use of a third-order term simultaneous with a second- and first-order term in the model led to low tolerance levels and subsequent variance inflation. However, although multicollinearity was most certainly present for the use of higher ordered terms in the same model, it did not affect statistical tests of significance because of the large sample sizes-therefore, we did not find it necessary to center any of the neighborhood-level predictors. Neighborhood immigrant orientation demonstrated a declining monotonic function such that the lowest ranges of immigrant orientation had the largest effect on the risk of low birth weight, whereas the right tail (high levels of immigrant orientation) had diminishing effects on low-birth-weight risk. On the other hand, a simple natural logarithm of neighborhood disadvantage was the best-fitting functional form and is consonant with findings of diminishing deleterious effects of neighborhood poverty (e.g., O'Campo et al. 1997).

\section{Statistical Model: Hierarchical Logistic Regression Model}

We used a random-intercept hierarchical logistic regression model to conduct our multivariate analyses (Snijders and Bosker 1999). ${ }^{11}$ This model accounted for the nonindependence of individuals residing in the same census tracts and 
accounts for the invariance of neighborhood-level predictors (i.e., disadvantage and acculturation) within tracts. We used the gllamm procedure in Stata (Volume 8) to conduct these analyses because the maximum-likelihood estimation algorithm (i.e., adaptive quadrature) has been shown to be superior to those used in other, more conventional multilevel programs (Rabe-Hesketh, Skrondal, and Pickles 2002; Rodriguez and Goldman 1995). The model is as follows:

$$
\log i t\left(P_{i j}\right)=\gamma_{00}+\sum_{h=1}^{p} \gamma_{h 0} x_{h i j}+\sum_{k=1}^{q} \gamma_{0 k} z_{k j}+\sum_{k=1}^{q} \sum_{h=1}^{p} \gamma_{h k} z_{k j} x_{h i j}+U_{0 j}+\sum_{h=1}^{p} U_{h j} x_{h i j}+R_{i j}
$$

where $\log i t(p)=\ln \left(\frac{p}{1-p}\right)$

This model estimates the log odds (logit) for the probability of being born low birth weight for a full set of predictors and covariates $\left(\gamma_{h}\right)$ for various values of $x$ for the $i^{\text {th }}$ person in the $j^{\text {th }}$ census tract (Snijders and Bosker 1999). Neighborhoodlevel predictors-disadvantage and (un)acculturation, in this case-are considered values of $z$ for the $j^{\text {th }}$ group and can be used to explain either average probabilities for low birth weight across neighborhoods or variation in slopes across neighborhoods (i.e., cross-level interactions). Although testing for random variation of coefficients (nativity in this case) between census tracts is also possible with this flexible model, our theory dictates that variation in nativity is a function of community-level immigrant orientation and disadvantage. Therefore, we assume a priori that there is nonrandom variation in our key predictor: nativity.

Models are built according to the hypotheses previously specified and are presented in Tables 3 through 5. In particular, tests of the first hypothesis are presented in Table 3, tests of the second hypothesis in Table 4, and tests for the third hypothesis in Table 5. Reference categories for each of the dummy variables are included in brackets. We will proceed sequentially through each of the results, beginning with Table 3.

\section{RESULTS}

Model 1 of Table 3 indicates an immigrant advantage such that the native born are 24 percent more likely to have infants born low birth weight than the foreign born. This relationship persists net of individual- and neighborhood-level controls (Model 5, Table 3). Furthermore, immigrant-oriented neighborhoods are protective of health such that infants born to mothers living in immigrant-oriented neighborhoods are less likely to be born low birth weight net of individual-level acculturation (nativity) and neighborhood disadvantage (Model 2, Table 3). ${ }^{12}$ As expected, neighborhood disadvantage has a deleterious effect on birth weight net of individual-level predictors (Model 3, Table 3). These effects persist net of individual-level nativity (Model 4, Table 3) and net of all individual-level controls in the model (Model 5, Table 3). Furthermore, it should be pointed out that the most typical risk factors for low birth weight are also found in our Los Angeles data; that is, results for other predictors are consistent with prior empirical studies.

Table 4 includes statistical tests for the moderating effects of neighborhood conditions on nativity. Model 1 tests the hypothesis that neighborhood immigrant 
TABLE 3

Hierarchical Logistic Regression Model of Low Birth Weight-Main Effects: Nativity Status and Neighborhood Characteristics

\begin{tabular}{|c|c|c|c|c|c|}
\hline Variables & Model 1 & Model 2 & Model 3 & Model 4 & Model 5 \\
\hline \multicolumn{6}{|l|}{ Tract-level variables } \\
\hline $\operatorname{Ln}($ disadvantage $)$ & & & $.2311^{* *}$ & $.3204^{* *}$ & $0.1732^{* *}$ \\
\hline Immigrant orientation $^{3}$ & & $.0112^{* *}$ & & $.0079^{* *}$ & $0.0081^{* *}$ \\
\hline$L n(\text { immigrant orientation })^{3}$ & & $-.0007^{* *}$ & & $-.0005^{* *}$ & $-0.0005^{* *}$ \\
\hline \multicolumn{6}{|l|}{ Individual-level variables } \\
\hline \multicolumn{6}{|l|}{ Nativity [native born] } \\
\hline Foreign born & $-.2154^{* *}$ & & & $-.2532^{* *}$ & $-0.0812^{*}$ \\
\hline Missing & .3936 & & & $.3989^{+}$ & $0.3952^{\dagger}$ \\
\hline Mother's age ${ }^{5}$ & & & & & $-2.5826^{* *}$ \\
\hline Mother's age & & & & & $0.2707^{* *}$ \\
\hline \multicolumn{6}{|l|}{ Parity [first birth] } \\
\hline Low parity & & & & & $-0.4279^{* *}$ \\
\hline High parity & & & & & $-0.3936^{* *}$ \\
\hline \multicolumn{6}{|l|}{ Marital status [unmarried] } \\
\hline Married & & & & & $-0.0736^{* *}$ \\
\hline \multicolumn{6}{|l|}{$\begin{array}{l}\text { Race/ethnicity [White, } \\
\text { non-Hispanic] }\end{array}$} \\
\hline Latino & & & & & -0.0316 \\
\hline Black, non-Hispanic & & & & & $0.6458^{* *}$ \\
\hline Other & & & & & 0.1447 \\
\hline Mother's education ${ }^{3}$ & & & & & $0.0006^{* *}$ \\
\hline Mother's education $^{3 *}$ & & & & & $-0.0002^{* *}$ \\
\hline $\operatorname{Ln}($ education) & & & & & \\
\hline \multicolumn{6}{|c|}{ Payer for birth [private insurance] } \\
\hline Government & & & & & $0.1112^{* *}$ \\
\hline Self-pay & & & & & $0.4180^{* *}$ \\
\hline Other & & & & & $0.4436^{+}$ \\
\hline \multicolumn{6}{|l|}{ Prenatal care [adequate] } \\
\hline Inadequate & & & & & $0.6264^{* *}$ \\
\hline Intermediate & & & & & -0.0569 \\
\hline Adequate plus & & & & & $1.1978^{* *}$ \\
\hline Missing/no care & & & & & $0.4142^{* *}$ \\
\hline \multicolumn{6}{|l|}{ Sex of child [female] } \\
\hline Male & & & & & $-0.1149^{* *}$ \\
\hline Constant & -2.8528 & -3.2329 & -3.5691 & -3.7508 & 2.0745 \\
\hline$N$ size & 140,472 & 140,472 & 140,472 & 140,472 & 140,472 \\
\hline Wald chi-square $(d f)$ & $80.71(2)$ & $20.81(3)$ & $91.13(1)$ & $238.61(6)$ & $2984.45(24)$ \\
\hline
\end{tabular}

$+p<.10 ;{ }^{*} p<.05 ;{ }^{* *} p<.01$.

orientation moderates nativity effects on the probability of low birth weight. Statistical results show a significant interaction term between immigrant orientation and nativity; plotting this relationship (Figure 1) demonstrates that this statistically significant product term indicates that residence with co-ethnics is much more protective of the foreign born, although it is modestly so for the native born. ${ }^{13}$ Model 2 
TABLE 4

Hierarchical Logistic Regression Model of Low Birth Weight-Interaction Effects: Nativity Status and Neighborhood Characteristics

\begin{tabular}{|c|c|c|}
\hline Variables & Model 1 & Model 2 \\
\hline \multicolumn{3}{|l|}{ Neighborhood-level variables } \\
\hline $\operatorname{Ln}($ disadvantage $)$ & $0.1748^{* *}$ & $0.2694^{* *}$ \\
\hline Immigrant orientation $^{3}$ & $0.0075^{* *}$ & $0.0066^{*}$ \\
\hline $\operatorname{Ln}(\text { immigrant orientation })^{3}$ & $-0.0038^{* *}$ & $-0.0034^{*}$ \\
\hline \multicolumn{3}{|l|}{ Individual-level variables } \\
\hline \multicolumn{3}{|l|}{ Nativity [native born] } \\
\hline Foreign born & 0.0029 & $0.4315^{* *}$ \\
\hline Missing & 0.4268 & -0.2164 \\
\hline Mother's age $\mathrm{e}^{5}$ & $-2.5342^{* *}$ & $-2.5253^{* *}$ \\
\hline Mother's age & $0.2664^{* *}$ & $0.2660^{* *}$ \\
\hline \multicolumn{3}{|l|}{ Parity [first birth] } \\
\hline Low parity & $-0.4293^{* *}$ & $-0.4311^{* *}$ \\
\hline High parity & $-0.3975^{* *}$ & $-0.4010^{* *}$ \\
\hline \multicolumn{3}{|l|}{ Marital status [unmarried] } \\
\hline Married & $-0.0724^{* *}$ & $-0.0700^{*}$ \\
\hline \multicolumn{3}{|l|}{ Race/ethnicity [White, non-Hispanic] } \\
\hline Latino & -0.0367 & -0.0248 \\
\hline Black, non-Hispanic & $0.6458^{* *}$ & $0.6092^{* *}$ \\
\hline Other & 0.1423 & 0.1382 \\
\hline Mother's education $^{3}$ & $0.0006^{* *}$ & $0.0005^{*}$ \\
\hline Mother's education ${ }^{3} \times \operatorname{Ln}($ Education $)$ & $-0.0002^{* *}$ & $-0.0002^{*}$ \\
\hline \multicolumn{3}{|l|}{ Payer for birth [private insurance] } \\
\hline Government & $0.1135^{* *}$ & $0.1152^{* *}$ \\
\hline Self-pay & $0.4160^{* *}$ & $0.4094^{* *}$ \\
\hline Other & $0.4460^{\dagger}$ & $0.4458^{\dagger}$ \\
\hline \multicolumn{3}{|l|}{ Prenatal care [adequate] } \\
\hline Inadequate & $0.6254^{* *}$ & $0.6271^{* *}$ \\
\hline Intermediate & -0.0565 & -0.0553 \\
\hline Adequate-plus & $1.1972^{* *}$ & $1.1989^{* *}$ \\
\hline Missing/no care & $0.4136^{* *}$ & $0.4184^{* *}$ \\
\hline \multicolumn{3}{|l|}{ Sex of child [female] } \\
\hline Male & $-0.1150^{* *}$ & $-0.1154^{* *}$ \\
\hline Disadvantage $\times$ Foreign Born & & $-0.2029^{* *}$ \\
\hline Disadvantage $\times$ Nativity Missing & & 0.2327 \\
\hline Acculturation $\times$ Foreign Born & $-0.0001^{* *}$ & \\
\hline Acculturation $\times$ Nativity Missing & -0.0001 & \\
\hline Constant & 1.9443 & 1.7403 \\
\hline$N$ & 140,472 & 140,472 \\
\hline Wald chi-square (df) & $2,992.31(26)$ & $3,001.09(26)$ \\
\hline
\end{tabular}

$+p<.10 ;{ }^{*} p<.05 ; * * p<.01$. 


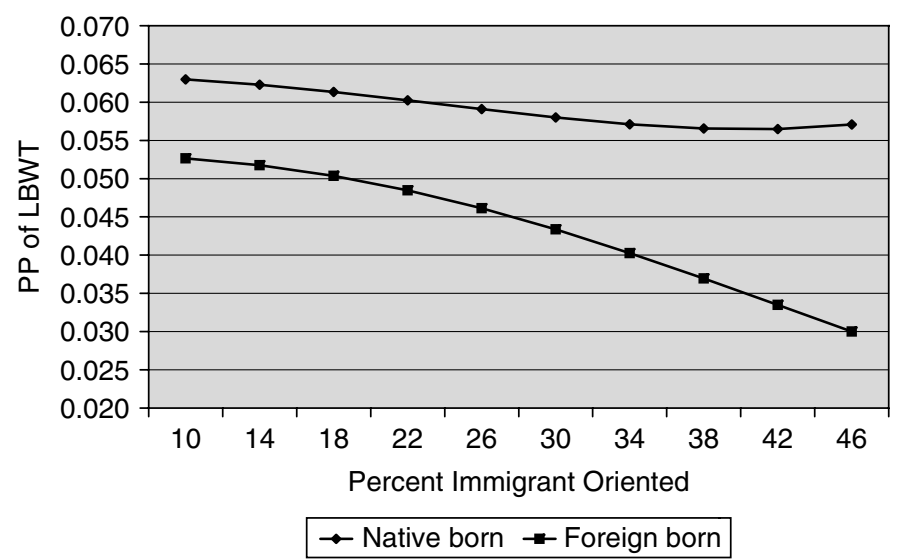

Figure 1

Predicted Probability of Low Birth Weight by Percent Immigrant Oriented in the Census Tract by Nativity Status

Note: $\mathrm{PP}=$ predicted probability; LBWT = low birth weight.

(see Table 4) predicts that neighborhood disadvantage also moderates the nativity effect on low birth weight, and plotting this relationship (Figure 2) demonstrates that this interaction is both statistically and substantively significant. In particular, the effects of living in a disadvantaged neighborhood are less deleterious to infants born to foreign-born women than they are to infants born to native-born women.

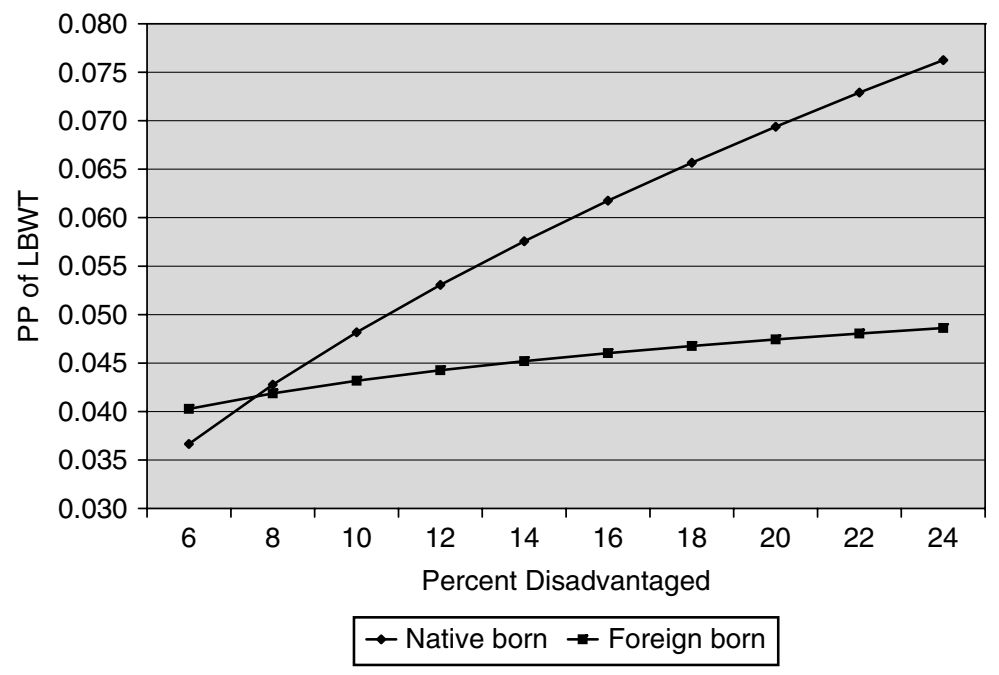

Figure 2

Predicted Probability for Low Birth Weight by Percent Disadvantaged in the Census Tract by Nativity Status

Note: PP = predicted probability; LBWT = low birth weight. 
Table 5 includes statistical tests for the individual effects of the more detailed measure of nativity as well as the potential moderating effects that the neighborhood might have on the risk of low birth weight. Model 1 of Table 5 predicts that labor migrants experience better birth outcomes than the native born, whereas professional migrants are similar to the native born. However, net of individuallevel controls, professional migrants experience worse outcomes than the native born (Model 2, Table 5). Interactions with neighborhood immigrant orientation (Model 3, Table 5) indicate that predicted risks for low birth weight converge for each of these groups in neighborhoods with high levels of immigrant orientation, whereas in low-immigrant-oriented neighborhoods, professional migrants are at highest risk and labor migrants at lowest risk of giving birth to a low-birthweight infant (see Figure 3). On the other hand, only the slope for labor migrants is statistically different from the native born (Model 3, Table 5). In addition, although professional migrants are most at risk and labor migrants least at risk, the slope for neighborhood disadvantage is largest among the native born and smallest among labor migrants (see Figure 4). Again, however, the slope for professional migrants is statistically different than that of the native born, whereas the slope for labor migrants is (statistically) equivalent to the native born (Model 4, Table 5).

\section{DISCUSSION AND CONCLUSIONS}

Using population-based data for all births in Los Angeles County in 2000, we were able to test expectations for a key health outcome, low birth weight, among individuals with heterogeneous characteristics. Adapting the theory of segmented assimilation-in which assimilation is construed as deleterious to health-we were able to posit three divergent pathways that immigrants might follow after immigration to the United States and assess the applicability of this theory to health outcomes in general and low birth weight in particular. In general, results confirm that interactions between individual markers for acculturation and community characteristics are crucial in understanding why immigrants' health is thought to deteriorate over time in the United States. We now compare our hypotheses with our empirical results.

As expected, infants of immigrants experience healthier birth weights (Hypothesis 1a), although this effect appears to be limited to labor migrants. In addition, immigrant-oriented neighborhoods are salutary (Hypothesis 1b), whereas disadvantaged neighborhoods increase the probability of giving birth to a low-birth-weight infant (Hypothesis 1c). Nonetheless, these main effect pathways have been generally well established in the public health and sociological literatures. Segmented assimilation theory offers a more nuanced expectation for these effects in a cross-level interaction framework, and we turn to these findings now.

Recent immigrants are hypothesized to have greater access to familial social support networks (Perez and Padilla 2000), be less likely to engage in risky behaviors during pregnancy (Finch et al. 2000), and be protected from health shocks by a host of characteristics indicative of native culture (Scribner and Dwyer 1989). It is 
TABLE 5

Hierarchical Logistic Regression Model of Low Birth Weight-Interaction Effects: Migrant Type by Neighborhood Characteristics

\begin{tabular}{|c|c|c|c|c|}
\hline Variables & Model 1 & Model 2 & Model 3 & Model 4 \\
\hline \multicolumn{5}{|l|}{ Neighborhood-level variables } \\
\hline $\operatorname{Ln}($ disadvantage $)$ & $0.3467^{* *}$ & $0.1727^{* *}$ & $0.1789^{* *}$ & $0.2258^{* *}$ \\
\hline Immigrant orientation $^{3}$ & $0.0075^{* *}$ & $0.0072^{* *}$ & $0.0069^{* *}$ & $0.0064^{*}$ \\
\hline $\operatorname{Ln}(\text { immigrant orientation })^{3}$ & $-0.0039^{* *}$ & $-0.0036^{* *}$ & $-0.0035^{* *}$ & $-0.0033^{*}$ \\
\hline \multicolumn{5}{|l|}{ Individual-level variables } \\
\hline \multicolumn{5}{|l|}{ Nativity [native born] } \\
\hline Labor migrant & $-0.2923^{* *}$ & $-0.1679^{* *}$ & $-0.1375^{* *}$ & 0.1479 \\
\hline Professional migrant & -0.0572 & $0.2258^{* *}$ & $0.3434^{* *}$ & $0.4132^{+}$ \\
\hline Missing & $0.3963^{\dagger}$ & $0.4252^{\dagger}$ & 0.4406 & -0.2254 \\
\hline Mother's age $^{5}$ & & $-2.4467^{* *}$ & $-2.4174^{* *}$ & $-2.4310^{* *}$ \\
\hline Mother's age & & $0.2595^{* *}$ & $0.2569^{* *}$ & $0.2582^{* *}$ \\
\hline \multicolumn{5}{|l|}{ Parity [first birth] } \\
\hline Low parity & & $-0.4353^{* *}$ & $-0.4363^{* *}$ & $-0.4361^{* *}$ \\
\hline High parity & & $-0.4092^{* *}$ & $-0.4122^{* *}$ & $-0.4113^{* *}$ \\
\hline \multicolumn{5}{|l|}{ Marital status [unmarried] } \\
\hline Married & & $-0.0689^{*}$ & $-0.0675^{*}$ & $-0.0676^{*}$ \\
\hline \multicolumn{5}{|l|}{ Race/ethnicity [White, non-Hispanic] } \\
\hline Latino & & -0.0084 & -0.0145 & -0.0073 \\
\hline Black, non-Hispanic & & $0.6432^{* *}$ & $0.6392^{* *}$ & $0.6232^{* *}$ \\
\hline Other & & 0.1483 & 0.1431 & 0.1437 \\
\hline Mother's education ${ }^{3}$ & & $0.0008^{* *}$ & $0.0008^{* *}$ & $0.0008^{* *}$ \\
\hline Mother's education ${ }^{3} \times \operatorname{Ln}($ education $)$ & & $-0.0003^{* *}$ & $-0.0003^{* *}$ & $-0.0003^{* *}$ \\
\hline \multicolumn{5}{|l|}{ Payer for birth [private insurance] } \\
\hline Government & & $0.1137^{* *}$ & $0.1160^{* *}$ & $0.1157^{* *}$ \\
\hline Self-pay & & $0.4040^{* *}$ & $0.4087^{* *}$ & $0.4009^{* *}$ \\
\hline Other & & $0.4432^{\dagger}$ & $0.4486^{\dagger}$ & $0.4444^{\dagger}$ \\
\hline \multicolumn{5}{|l|}{ Prenatal care [adequate] } \\
\hline Inadequate & & $0.6242^{* *}$ & $0.6241^{* *}$ & $0.6249^{* *}$ \\
\hline Intermediate & & -0.0572 & -0.0566 & -0.0562 \\
\hline Adequate-plus & & $1.1996^{* *}$ & $1.1989^{* *}$ & $1.2001^{* *}$ \\
\hline Missing/no care & & $0.4140^{* *}$ & $0.4130^{* *}$ & $0.4167^{* *}$ \\
\hline \multicolumn{5}{|l|}{ Sex of child [female] } \\
\hline Male & & $-0.1149^{* *}$ & $-0.1148^{* *}$ & $-0.1152^{* *}$ \\
\hline Disadvantage $\times$ Labor Migrant & & & & $-0.1203^{*}$ \\
\hline Disadvantage $\times$ Professional Migrant & & & & -0.0853 \\
\hline Disadvantage $\times$ Nativity Missing & & & & 0.2482 \\
\hline Acculturation $\times$ Labor Migrant & & & -0.0000 & \\
\hline Acculturation $\times$ Professional Migrant & & & $-0.0002^{*}$ & \\
\hline Acculturation × Nativity Missing & & & -0.0000 & \\
\hline Constant & -3.8102 & 1.7514 & 1.6430 & 1.6078 \\
\hline$N$ & 140,472 & 140,472 & 140,472 & 140,472 \\
\hline Wald chi-square $(d f)$ & $260.59(7)$ & $3,015.84(25)$ & $3,021.89(28)$ & $3,021.62(28)$ \\
\hline
\end{tabular}

$+p<.10 ;{ }^{*} p<.05 ;{ }^{* *} p<.01$. 


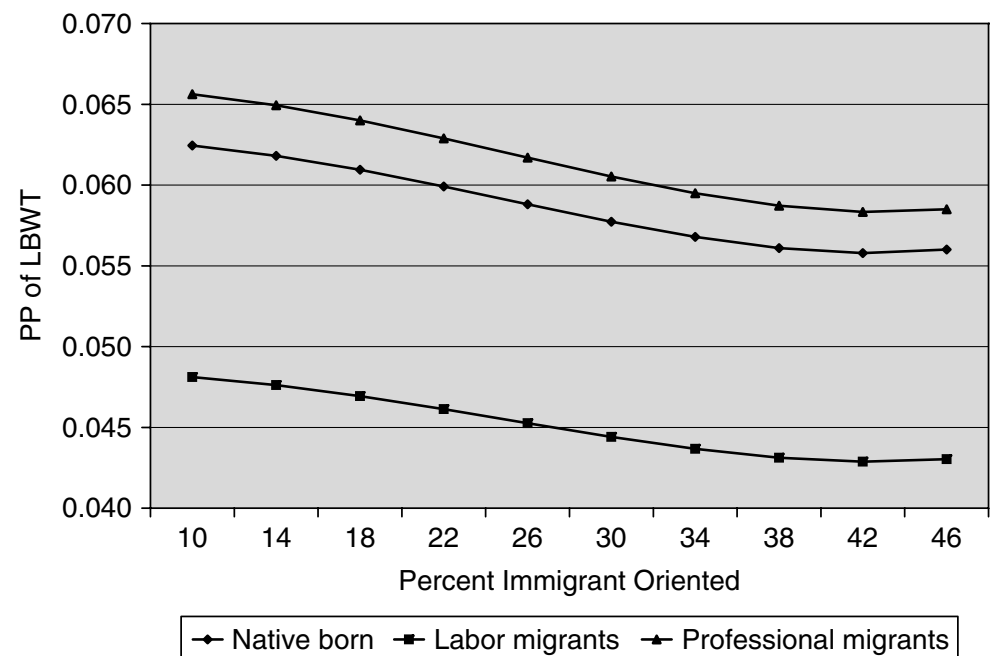

Figure 3

Predicted Probability of Low Birth Weight by Percent Immigrant Oriented in the Census Tract by Migrant Type

Note: $\mathrm{PP}=$ predicted probability; LBWT =low birth weight.

expected that these protective factors may erode over time in the United States such that the native born (second generation) are less culturally distinct from individuals with long family tenures in the United States. Our adapted theory suggests that the foreign born may be more able to draw on these protective resources from the community than the foreign born, at least partially because of potential primary

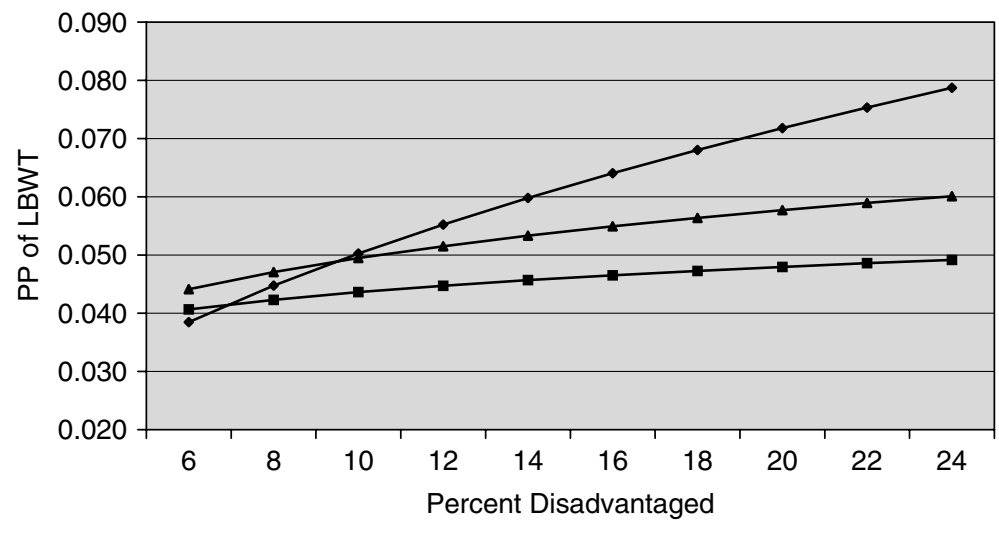

Native born $\rightarrow$ - Labor migrants $\rightarrow$ Professional migrants

Figure 4

Predicted Probability for Low Birth Weight by Percent Disadvantaged in the Census Tract by Migrant Type

Note: $\mathrm{PP}=$ predicted probability; LBWT = low birth weight. 
language differences (Hypothesis 2a). However, aside from a slight divergence at moderately high levels of acculturation (e.g., 14 to 30 percent), immigrant-oriented neighborhoods are universally protective of health net of the levels of poverty in the neighborhood (i.e., neighborhood disadvantage). Similarly, we expect that foreign-born women may be less likely to engage in the maladaptive coping strategies of the underclass than the native born because of language and cultural differences and because of the higher probability for cultural protectiveness to be intact (Hypothesis $2 b$ ). Our findings generally confirm this result given that increasingly disadvantaged neighborhoods are more harmful to the native born than they are to the foreign born.

To reiterate, the predicted immigrant health advantage applies largely to labor migrants (Hypothesis 3a). Furthermore, given that professional migrants may be more upwardly mobile and have more chances for interaction with adherents to mainstream U.S. culture (residentially and through the workplace), it is expected that they will either be marginalized from, or draw less on, community resources available in immigrant-oriented neighborhoods. Our empirical results confirm this, such that non-immigrant-oriented neighborhoods are especially harmful to professional migrants-although the effect of neighborhood acculturation among labor migrants is statistically indistinguishable from the effect among the native born (Hypothesis $3 b$ ). In other words, professional immigrants do appear to adapt more readily to mainstream norms, to the detriment of their health. Finally, although neighborhood disadvantage is universally deleterious to birth outcomes, it is less harmful among labor migrants than it is for professional migrants and the native born (Hypothesis 3c). This result indicates that low-skilled migrants are at least partially inured to the effects of disadvantaged neighborhoods or are able to draw on cultural resources to cope with the shocks associated with living in more impoverished neighborhoods. The fact that native born infants are more likely to be born low birth weight in disadvantaged neighborhoods provides indirect evidence that adaptation to underclass neighborhoods may be harming immigrant health further.

Therefore, although individual characteristics and choices surely help to determine the ways in which infant health is produced, structural factors play a role as well. From these analyses, the evidence suggests that residence in immigrant enclaves may stave off assimilation while simultaneously preserving good health. On the contrary, immigrants who settle in highly acculturated neighborhoods may assimilate more rapidly and erode any of the original health protectiveness that native cultures may have offered. In short, by adapting the theory of segmented assimilation to health, we are able to identify some of the broader, sociodemographic patterns through which individuals may come to assimilate and the effect this may have on their health. Most importantly, we confirm that health trajectories among immigrants are highly divergent over time and that overall health declines are not inevitable; in fact, health maintenance was observed among immigrant women who coresided in heavily immigrant neighborhoods. This should come as good news for public health agencies and provide a ripe field of study for public health researchers. 


\section{Limitations}

There are several limitations to our study worth discussing. First, what we gain in sample size and the sheer ability to test differences across a wide range of neighborhoods, we lose in terms of the complexity of our measures. Thus, although empirical results using measures of nativity and tract-level acculturation are certainly consistent with the core of segmented assimilation theory, suggested key variables, and theoretical expectations for effects, more nuanced measures of acculturation, ethnic identity, and immigrant enclaves may yield richer results. Absent this type of data, however, this is currently not an option. Second, these results may be unique to birth outcomes and women, as are several findings related to the epidemiological paradox (Palloni and Morenoff 2002). Third, the use of administrative boundaries (i.e., census tracts) to represent one's range of contacts and acculturative context may be supplanted by self-defined interactional ranges in the future; again, however, current data collection efforts may not overcome this problem. On the other hand, self-defined and self-described neighborhoods may simply reflect response bias that is the result of compositional differences between neighborhood respondents rather than true neighborhood differences (Sampson, Raudenbush, and Earls 1997).

Fourth, observed neighborhood effects may be endogenous to individual preferences and levels of acculturation such that observed effects may simply be the result of unmeasured levels of individual acculturation and/or neighborhood choice. Nonetheless, although this would dramatically change future approaches to testing the segmented assimilation theory, it still provides support for the acculturation hypothesis in the public health literatures (i.e., that health declines among immigrants are associated with increasing acculturation). ${ }^{14}$ Finally, our ability to distinguish between labor and professional migrants is limited by the use of current levels of education. That is, we do not have a way of determining the level of education that one possessed at the time of immigration, and therefore, the predicted effects of professional migrants (i.e., worse birth outcomes, further amplified by contextual risk factors) may actually be effects for highly upwardly mobile labor migrants.

\section{Future Research}

Future research should test these processes using various populations, focusing particularly on adaptive strategies among first- and second-generation adolescents. In addition, more nuanced measures of assimilation may uncover more detailed pathways toward health declines and maintenance. For example, it may be that individuals with stronger ethnic identities may resist assimilation to a degree not anticipated by the use of simple nativity contrasts. Finally, the specific health behaviors, types of native social support, and pregnancy-specific practices should be included in longitudinal studies to determine the actual pathways through which these cross-sectional associations between assimilation and health may be working.

In conclusion, the theory of segmented assimilation is particularly suitable for adaptation to health outcomes, bearing in mind the paradox of assimilation with 
regard to cultural adaptation and its health consequences. Adoption of cultural norms is inherently linked to ultimate expectations regarding the processes of socioeconomic mobility. Therefore, although assimilation may bring with it its share of both potential upward mobility and discontents, assimilation may be utterly deleterious to immigrant health.

\section{NOTES}

1. Much of the health literatures cited in this article are specific to Latinos in general and/ or Latino subgroups; however, recent (yet sparse) research indicates an overall immigrant advantage and subsequent decline in health with acculturation. Therefore, although much of the cited literature may be for Latino groups, we posit that similar effects exist for most immigrant groups.

2. This theory is formulated with the explicit intent of explaining disparate outcomes among immigrants and is therefore appropriately titled given that it is specified as the endpoint to acculturative and structural processes. However, this theory specifies that both individual acculturative characteristics and contextual/residential characteristics will partially determine the assimilation endpoints. Although acculturation may not be appropriately construed as a linear process, cultural orientation of the country of origin generally declines through generational processes such that second-generation immigrants are significantly less attached to cultural beliefs of the country of origin than are first-generation immigrants (Cuellar, Nyberg, and Maldonado 1997; Perez and Padilla 2000). Therefore, although it is not our intent to describe the health endpoints for these individuals-given that health is a dynamic process and measure-it is our intent to demonstrate that some of the individual and structural processes at play will at least partially predict the health profiles of immigrants.

3. These data are only available through contract with the Los Angeles County Department of Health Services and are not available from the corresponding author. However, we will gladly refer inquiring researchers to the most direct contact and provide researchers with appropriate program files if requested.

4. Although the public data records contain the census tract of residence for each birth mother, the data were geo-coded to 1990 census tracts and were based on old address files, which lead to a high rate of missing and incorrect tract numbers.

5. These exclusions are split between individuals with higher socioeconomic status (excluded because their census tracts are not available in current address files, as they are largely in new suburban developments) and individuals with lower socioeconomic status for whom birth certificate data (length of gestation and birth weight) were erroneously recorded.

6. Although the majority of our variables are categorical in nature and hence represented by dummy variables in each regression model, a few variables are measured continuously and several tests for various functional forms are considered to improve model fit and specification. In particular, we employ a fractional polynomial regression approach (covariate adjusted) to determine the best functional form for each continuous variable (Royston and Altman 1994). In some instances, this approach led to implausible functional forms, and those most consonant with current epidemiological knowledge on the relationships were chosen over higher ordered terms that provided a slightly better model fit. 
7. Although birth weight is determined by both gestational age at delivery and the fetal growth rate, low-birth-weight infants account for approximately two-thirds of the nation's neonatal deaths (Kiely et al. 1994).

8. Parity is defined as high in third or higher numbered births to women less than twenty-five years old and fourth or higher numbered births to women twenty-five to twenty-nine years old. Primiparous births are first births, and all other births are considered low parity.

9. Other approaches included creating a standardized score from various factor analysis specifications, but the presence of negative values in this factor score (i.e., those below the mean) obviated the use of fractional polynomials that relied on logarithmic conversions. As with age and education, we employed a fractional polynomial regression approach combined with plausible functional shapes for these relationships to decide on the most accurate functional form.

10. The census defines a linguistically isolated household as one in which no person age fourteen or older speaks only English and no person age fourteen or older who speaks a different language other than English speaks English very well.

11. A logit model is also preferable to a probit model, as our dependent variable is a relatively rare event (just under 5 percent of the cases are low birth weight). In short, the logit transformation is more sensitive to covariate effects at the tails of this distribution.

12. Predicted probabilities are plotted only for the 10th to the 90th percentiles of the neighborhood distributions and therefore do not project outside the observed range of the date.

13. For ease of presentation, we will occasionally refer to a highly unacculturated neighborhood as an "ethnic enclave," although we do not select any arbitrary cut point at which we expect this qualitative change.

14. On the other hand, if neighborhood residence is simply a proxy for duration, for example, then these results may simply indicate health attrition over time rather than acculturation effects. This would be the case if more acculturated individuals moved out of enclaves and into neighborhoods with more members of the mainstream culture. Cross-tabulations indicate that foreign-born women are nominally older than native-born women (around one-half year) and that professional migrants are (on average) four years older than both labor migrants and the native born. Additionally, women are only slightly older (less than three years) in more acculturated neighborhoods. It is doubtful that these differences in age would yield tremendous differences in levels of acculturation. In addition, age is controlled for in the multivariate regression models.

\section{REFERENCES}

Alba, Richard and Victor Nee. 1999. "Rethinking Assimilation Theory for a New Era of Immigration." Pp. 137-60 in The Handbook of International Migration: The American Experience, edited by C. Hirschman, P. Kasinitz, and J. DeWind. New York: Russell Sage Foundation.

Angel, Ronald and Peter J. Guarnaccia. 1989. "Mind, Body, and Culture: Somatization among Hispanics." Social Science and Medicine 28(12): 1229-38.

$\rightarrow$ Barker, David J. 1995. "Fetal Origins of Coronary Heart Disease." British Journal of Medicine 311: 171-74. 
$\rightarrow$ Boardman, Jason D., Brian K. Finch, and Robert A. Hummer. 2001. "Race/Ethnic Differences in Respiratory Problems among a Nationally Representative Cohort of Young Children in the United States." Population Research and Policy Review 20: 187-206.

Boardman, Jason D., Daniel A. Powers, Yolanda C. Padilla, and Robert A. Hummer. 2002. "Low Birth Weight, Social Factors, and Developmental Outcomes among Children in the United States." Demography 39(2): 353-68.

Card, David, John DiNardo, and Eugena Estes. 2000. "The More Things Change: Immigrants and the Children of Immigrants in the 1940s, and the 1970s, and the 1990s." Pp. 227-70 in Issues in the Economics of Immigration, edited by G. Borjas. Chicago: University of Chicago Press.

Collins, James W. and David K. Shay. 1994. "Prevalence of Low Birth Weight among Hispanic Infants with United States-Born and Foreign-Born Mothers: The Effect of Urban Poverty." American Journal of Epidemiology 139(2): 184-92.

Cuellar, Israel, Bill Nyberg, and Roberto E. Moldonado. 1997. "Ethnic Identity and Acculturation in a Young Adult Mexican-Origin Population." Journal of Community Psychology 25(6): 535-49.

$\rightarrow$ Elo, Irma T. and Samuel H. Preston. 1992. "Effects of Early-Life Conditions on Adult Mortality: A Review." Population Index 58: 186-212.

Finch, Brian K., Jason D. Boardman, Bohdan Kolody, and William A. Vega. 2000. “Contextual Effects of Acculturation on Perinatal Substance Exposure among Immigrant and Native-Born Latinas." Social Science Quarterly 81(1): 459-76.

$\rightarrow$ Finch, Brian K., Robert A. Hummer, Maureen Reindl, and William A. Vega. 2002. "The Validity of Self-Rated Health among Latino(a)s." American Journal of Epidemiology 155(8): 755-59.

Frisbie, W. Parker, Youngtae Cho, and Robert A. Hummer. 2001. "Immigration and the Health of Asian and Pacific Islander Adults in the United States." American Journal of Epidemiology 153(4): 372-80.

$\rightarrow$ Gortmaker, Stephen L. and Paul Wise. 1997. “The First Injustice: Socioeconomic Disparities, Health Services Technology, and Infant Mortality." Annual Review of Sociology 23: 147-70.

Guendelman, Sylvia. 1998. "Immigrants May Hold Clues to Protecting Health During Pregnancy: Exploring a Paradox." Pp. 222-57 in Promoting Human Wellness: New Frontiers for Research, Policy, and Practice, edited by M. Schneider-Jamner and D. Stokols. Berkeley: University of California Press.

$\rightarrow$ Guendelman, Sylvia, Pierre Buekens, Beatrice Blondel, Monique Kaminski, Francis C. Notzon, and Godelieve Masuy-Stroobant. 1999. "Birth Outcomes of Immigrant Women in the United States, France, and Belgium." Maternal and Child Health Journal 3 (4): $177-87$.

$\rightarrow$ Hack, Maureen, Nancy K. Klein, and H. Gerry Taylor. 1995. “Long-Term Developmental Outcomes of Low Birth Weight Infants." The Future of Children 5: 176-96.

$\rightarrow$ Harker, Kathryn. 2001. "Immigrant Generation, Assimilation, and Adolescent Psychological Well-Being." Social Forces 79: 969-1004.

$\rightarrow$ Hummer, Robert, Monique Biegler, Peter DeTurk, Douglas Forbes, W. Parker Frisbie, Ying Hong, and Starling Pullum. 1999. "Race/Ethnicity, Nativity, and Infant Mortality in the United States." Social Forces 77(3): 1083-118.

Hummer, Robert, Richard Rogers, Charles Nam, and Felicia LeClere. 1999. "Race/Ethnicity, Nativity, and U.S. Adult Mortality." Social Science Quarterly 80(1): 136-53.

Kiely, John L., Kate M. Brett, Stella Yu, and Diane L. Rowley. 1994. “Low Birth Weight and Intrauterine Growth Retardation." Pp. 185-202 in From Data to Action: CDC's Public Health Surveillance for Women, Infants, and Children, edited by L. S. Wilcox. Atlanta, GA: Centers for Disease Control and Prevention. 
$\rightarrow$ Kleinman, Joel C. and Samuel S. Kessel. 1987. "Racial Differences in Low Birth Weight: Trends and Risk Factors." New England Journal of Medicine 317(12): 749-53.

$\rightarrow$ Kotelchuck, Milton. 1994. "The Adequacy of Prenatal Care Utilization Index: Its U.S. Distribution and Association with Low Birth-Weight." American Journal of Public Health 84(9): 1486-88.

$\rightarrow$ Markides, Kyriakos S. and Jeannine Coreil. 1986. "The Health of Hispanics in the Southwestern United States: An Epidemiologic Paradox." Public Health Reports 101(3): 253-65.

$\rightarrow$ Marmot, M, A. Adelstein, and L. Bulusu. 1984. "Lessons from the Study of Immigrants' Mortality." Lancet 1: 1455-57.

$\rightarrow$ McCormick, Marie C., Steven L. Gortmaker, and Arthur M. Sobol. 1990. “Very Low Birth Weight Children: Behavior Problems and School Difficulty in a National Sample." Journal of Pediatrics 117: 687-93.

$\rightarrow$ O'Campo, Patricia, Xiaonan Xue, Mei-Cheng Wang, and Margaret O'Brien Caughy. 1997. “Neighborhood Risk Factors for Low Birth-Weight in Baltimore: A Multilevel Analysis." American Journal of Public Health 87(7): 1113-18.

$\rightarrow$ Palloni, Alberto and Jeffrey D. Morenoff. 2002. "Interpreting the Paradoxical in the Hispanic Paradox: Demographic and Epidemiologic Approaches." Annals of the New York Academy of Sciences 954: 140-74.

$\rightarrow$ Perez, William and Amado M. Padilla. 2000. "Cultural Orientation across Three Generations of Hispanic Adolescents." Hispanic Journal of Behavioral Sciences 22(3): 390-98.

Portes, Alejandro and Rubén G. Rumbaut. 2001. Legacies: The Story of the Immigrant Second Generation. Berkeley: University of California Press.

$\rightarrow$ Portes, Alejandro and Min Zhou. 1993. "The New Second Generation: Segmented Assimilation and its Variants." Annals of the American Academy of Political and Social Sciences 539: 74-96.

Rabe-Hesketh, Sophia, Anders Skrondal, and Andrew Pickles. 2002. "Reliable Estimation of Generalized Linear Mixed Models Using Adaptive Quadrature." The Stata Journal 2(1): 1-21.

Rodriguez, B. and Noreen Goldman. 1995. "An Assessment of Estimation Procedures for Multilevel Models with Binary Responses." Journal of the Royal Statistical Society (Series A) 164: 339-55.

$\rightarrow$ Royston, Patrick and Douglas G. Altman. 1994. "Regression Using Fractional Polynomials of Continuous Covariates: Parsimonious Parametric Modelling (with Discussion)." Applied Statistics 43: 429-67.

$\rightarrow$ Rumbaut, Rubén G. 1997. "Assimilation and its Discontents: Between Rhetoric and Reality." International Migration Review 31(4): 923-60.

— and Kenji Ima. 1988. The Adaptation of Southeast Asian Refugee Youth: A Comparative Study. Washington, DC: Office of Refugee Resettlement.

_ and John R. Weeks. 1996. "Unraveling a Public Health Enigma: Why Do Immigrants Experience Superior Perinatal Health Outcomes?" Research in the Sociology of Health Care 13: 337-91.

Sampson, Robert J., Stephen W. Raudenbush, and Felton Earls. 1997. "Neighborhoods and Violent Crime: A Multilevel Study of Collective Efficacy." Science 277: 918-24.

Scribner, Richard. 1996. "Paradox as Paradigm-The Health Outcomes of Mexican Americans." American Journal of Public Health 86: 303-5.

$\rightarrow-$ and James H. Dwyer. 1989. "Acculturation and Low Birth-Weight among Latinos in the Hispanic HANES." American Journal of Public Health 79: 1263-67.

Snijders, Tom and Roel J. Bosker. 1999. Multilevel Analysis: An Introduction to Basic and Advanced Multilevel Modeling. Thousand Oaks, CA: Sage. 
$\rightarrow$ Sorlie, P. D., E. Backlund, and N. J. Johnson. 1993. "Mortality by Hispanic Status in the United States." Journal of the American Medical Association 270: 2464-68.

UNICEF. 1996. The State of the World's Children. Oxford, UK: Oxford University Press.

$\rightarrow$ Vega, William A. and Hortensia Amaro. 1994. "Latino Outlook: Good Health, Uncertain Prognosis." Annual Review of Public Health 15: 39-67.

Wanner, P., M. Khlat, and C. Bouchardy. 1995. "Health Habits and Behavior of Southern European and North African Immigrants in France." Revue Epidemiologie et Sante Publique 43: 548-59. 